\title{
Where and When to Pay Attention: The Neural Systems for Directing Attention to Spatial Locations and to Time Intervals as Revealed by Both PET and fMRI
}

\author{
Jennifer T. Coull ${ }^{1}$ and Anna C. Nobre ${ }^{1,2}$ \\ ${ }^{1}$ Functional Imaging Laboratory, Wellcome Department of Cognitive Neurology, Institute of Neurology, London WC1N \\ 3BG, United Kingdom, and 2University of Oxford, Department of Experimental Psychology, Oxford OX1 3UD, \\ United Kingdom
}

Although attention is distributed across time as well as space, the temporal allocation of attention has been less well researched than its spatial counterpart. A temporal analog of the covert spatial orientation task [Posner MI, Snyder CRR, Davidson BJ (1980) Attention and the detection of signals. J Exp Psychol Gen 109:160-174] was developed to compare the neural systems involved in directing attention to spatial locations versus time intervals. We asked whether there exists a general system for allocating attentional resources, independent of stimulus dimension, or whether functionally specialized brain regions are recruited for directing attention toward spatial versus temporal aspects of the environment. We measured brain activity in seven healthy volunteers by using positron emission tomography (PET) and in eight healthy volunteers by using functional magnetic resonance imaging (fMRI). The task manipulated cued attention to spatial locations (S) and temporal intervals $(T)$ in a factorial design. Symbolic central cues oriented subjects toward S only (left or right), toward T only (300 msec or $1500 \mathrm{msec}$ ), toward both $\mathrm{S}$ and $\mathrm{T}$ simultaneously, or provided no information regarding $\mathrm{S}$ or $\mathrm{T}$. Subjects also were scanned during a resting baseline condition. Behavioral data showed benefits and costs for performance during temporal attention similar to those established for spatial attention. Brain-imaging data revealed a partial overlap between neural systems involved in the performance of spatial versus temporal orientation of attention tasks. Additionally, hemispheric asymmetries revealed preferential right and left parietal activation for spatial and temporal attention, respectively. Parietal cortex was activated bilaterally by attending to both dimensions simultaneously. This is the first direct comparison of the neural correlates of attending to spatial versus temporal cues.

Key words: attention; space; time; orienting; parietal; lateralization; imaging
Imagine waiting at traffic lights in London that have just turned from red to amber. With this cue, anticipation of the moment when the lights turn green begins. Common experience tells us that focusing attention on the anticipated moment will lead to quicker responses than if our mind is occupied elsewhere. Similarly, within the spatial domain, attention must be directed toward the light to respond efficiently. Focusing of attention within the spatial domain has been well investigated. The brain is capable of directing attention dynamically across extrapersonal space on the basis of expectations of where relevant events are likely to appear. Stimuli appearing in predicted locations are detected more rapidly and accurately than those that are not (Posner et al., 1980). Convergent findings from neuropsychological (for review, see Mesulam, 1990) and brain-imaging studies (Corbetta et al., 1993; Nobre et al., 1997) have defined the neural network for spatial attention, the core elements of which are posterior parietal cortex around intraparietal sulcus, frontal eye fields in lateral and medial premotor cortex, anterior cingulate, and subcortical areas.

Received March 25, 1998; revised June 29, 1998; accepted June 30, 1998.

J.T.C. was funded by the Medical Research Council during the acquisition of these data and is now funded by The Wellcome Trust. The experiments were partially supported by a project grant to A.C.N. by The Wellcome Trust. We thank Professors Dick Passingham and Chris Frith for invaluable discussion and comments, and Dr. Christian Buechel for advice on analysis of fMRI data.

Correspondence should be addressed to Drs. J.T. Coull or A.C. Nobre, Functional Imaging Laboratory, Wellcome Department of Cognitive Neurology, Institute of Neurology, 12 Queen Square, London WC1N 3BG, UK.

Copyright (C) 1998 Society for Neuroscience $\quad 0270-6474 / 98 / 187426-10 \$ 05.00 / 0$
By contrast, there has been little or no research investigating attentional orienting to a particular point in time (but see Kingstone, 1992). Selective temporal attention is distinct from vigilance and from the "attentional blink" in which target items occurring in close temporal proximity compete for limited processing resources (Raymond et al., 1992). Attentional orienting in time concerns whether and how information about time intervals can be used to direct attention to a point in time when a relevant event is expected, to optimize behavior. Temporal orienting will depend on elementary time perception processes, the neural correlates of which include the cerebellum (Ivry and Keele, 1989; Jueptner et al., 1995; Nichelli et al., 1996); basal ganglia (Rao et al., 1997; Harrington et al., 1998), and frontal cortex (Nichelli et al., 1995; Harrington et al., 1998).

Our aim is to reveal the brain regions involved in directing attention toward a particular time point once the time interval has been estimated. Furthermore, we investigate the anatomical overlap between the neural systems for spatial and temporal attention. We developed a temporal analog of the spatial orienting of attention task (Posner, 1980). In this task the subjects respond as quickly as possible to visual targets appearing at peripheral locations. Immediately preceding the target is a visual cue that either correctly ("valid cue") or incorrectly ("invalid cue") predicts the location of the upcoming target. Similarly, in our temporal orienting task, we assessed whether stimuli that occurred at predictable cued intervals were detected more efficiently than those that did not occur at the predicted moment. Brain imaging with PET 
and fMRI was used to visualize the neural system involved in directing attention across time, which was compared directly with that obtained during spatial orienting. We ask whether there is a unitary system for allocating limited-capacity attentional resources that is independent of the stimulus dimension used to direct expectancies or whether functionally specialized brain regions differentially deploy these resources toward aspects of the extrapersonal world.

\section{MATERIALS AND METHODS}

\section{Subjects}

Seven healthy right-handed male volunteers (mean age 29) took part in the PET experiment. Eight healthy right-handed volunteers (mean age 30; 4 male, 4 female) took part in the fMRI experiment. Subjects were physically fit, and none were taking medication. The study was approved by the local hospital ethics committee, and written informed consent was obtained before the study.

\section{Experimental task}

The basic visual display consisted of a central cueing stimulus $\left(1^{\circ}\right.$ eccentricity) and two peripheral boxes ( $7^{\circ}$ eccentricity) inside which the target ("x" or "+") appeared. The subjects' task was to detect covertly the peripheral target stimuli as rapidly as possible while avoiding mistakes. No discrimination was required. The reason for using two types of stimulus was to enable compatibility with future studies in which stimulus discriminations are involved.

The task manipulated subjects' expectations of where or when target stimuli would appear within an experimental display. A $2 \times 2$ factorial design was used in which the experimental factors were spatial cueing $(\mathrm{S})$ and temporal cueing (T). Across runs there were four types of cues that predicted both spatial location and onset time (ST), target location only (S), target onset time only ( $\mathrm{T})$, and neutral cues that predicted neither target location nor onset time $(\mathrm{N})$.

The central cue was a compound stimulus consisting of a diamond and two concentric circles. One part of the cue highlighted to inform the subject whether to attend to the position of the target (left or right) or to the time of the target (300 or $1500 \mathrm{msec}$ from cue presentation) (Fig. 1a). During the S condition the left (or right) side of the diamond brightened to inform the subject that the target is likely to appear in the left (or right) peripheral box. During the T condition a brightening of the inner circle indicated that the target would appear within a short time interval $(300 \mathrm{msec})$, whereas a brightening of the outer circle represented a longer time interval $(1500 \mathrm{msec})$. During the ST condition one of the circles and one side of the diamond brightened, indicating one of four combinations of spatial location and temporal interval (see Fig. 1a). During the neutral cue $(\mathrm{N})$ condition the entire cue brightened, providing no spatial or temporal information.

The beginning of a trial was indicated by the brightening $(100 \mathrm{msec})$ of part of the central cue (Fig. 1b). Targets appeared for $50 \mathrm{msec}$ in one of the two peripheral boxes and at one of two time periods, according to the nature of the cue. Subjects indicated covert detection of the target by pressing a response button with their right index finger. The computer recorded reaction times (RTs) to target stimuli.

Subjects performed two behavioral experimental sessions, one before and one during the brain-imaging session. The behavioral session that preceded scanning familiarized the subjects with the task and measured each subject's attentional effects across experimental conditions. Two hundred trials were presented for each of the four experimental conditions. The proportion of trials with valid cues in the focused attention conditions (ST, S, T) was $80 \%$. Presentation order was randomized both within and between subjects. During the PET session each experimental run contained 60 trials and lasted $\sim 2.5 \mathrm{~min}$. Subjects also were scanned during a resting baseline condition in which they simply fixated on the static background display, which contained cue, peripheral target boxes, and targets. There was an overall ratio of 80:20 valid/invalid trials. During fMRI scanning, experimental conditions were presented successively and in alternation with the resting baseline condition. Only valid trials were imaged in the fMRI scanning session.

\section{PET scanning}

Scans of the distribution of perfusion were obtained for each subject, using a Siemens/CPS ECAT EXACT HR+ (model 962) PET scanner, (a)

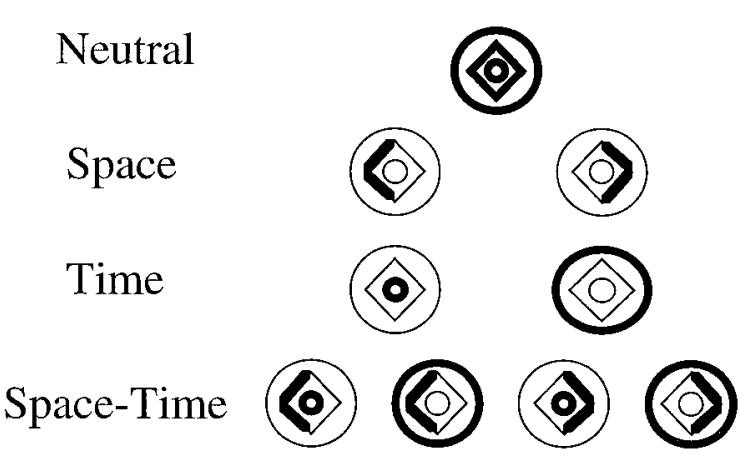

(b)

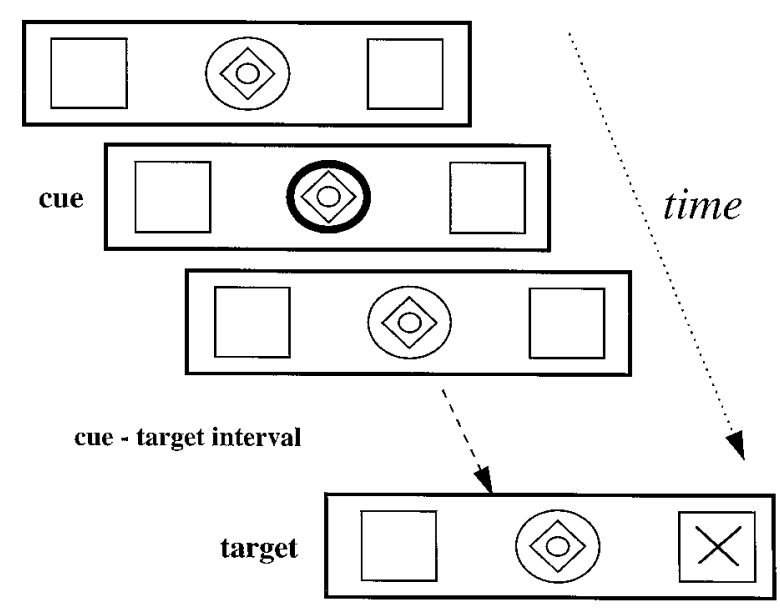

Figure 1. a, Attentional cues used to direct subjects' attention to a particular target location or stimulus-onset time. The neutral cue provides neither spatial nor temporal information, the spatial (Space) cue directs the subjects' attention to the left or right, the temporal (Time) cue directs attention to a short or long stimulus-onset time, and the spatiotemporal (Space-Time) cue directs attention to both location and stimulus-onset time. $b$, A typical trial, which in this example directs the subjects' attention to a long stimulus-onset time, with no information about the target's location. The attentional cue is on for $100 \mathrm{msec}$, the cue-target interval is either 300 or $1500 \mathrm{msec}$ (short/long cue), and finally the target appears for $50 \mathrm{msec}$ in either the left or right box.

with septa retracted, to collect data in a three-dimensional mode. A shielding insert was positioned at the front of the scanner to attenuate the contribution of radioactivity from the rest of the body to the recorded images. Radioactivity was administered as $\mathrm{a}_{2}{ }^{15} \mathrm{O}$ bolus, infused over $20 \mathrm{sec}$, followed by a $20 \mathrm{sec}$ saline flush. The total effective dose equivalent of radioactivity per subject was $5.0 \mathrm{mSv}$. Twelve PET emission scans were collected over $96 \mathrm{~min}$, with an 8 min interval between scans. Integrated radioactivity counts over a $90 \mathrm{sec}$ acquisition period, beginning with the rising phase of radioactivity in the head, were used as an index of local blood perfusion. A transmission scan was collected before emission scans to check the position of subjects and to correct for attenuation effects. Structural brain images also were obtained for each subject, using T1-weighted MRI to coregister significant functional PET activations with anatomy.

Of the 12 PET images that were obtained, 10 are relevant to the present report. Two functional PET images were obtained for each of the four experimental conditions of interest (ST, T, S, N). In addition, two images were obtained during a resting baseline condition. Brain images were collected after the subjects had been engaged in the task for $1 \mathrm{~min}$. The order in which conditions were presented was randomized both within and between subjects. 


\section{fMRI scanning}

Scans were acquired by using a 2 Tesla Magnetom Vision (Siemens, Erlangen, Germany) whole-body MRI system, which was equipped with a head coil. Echo-planar imaging (EPI) was used to obtain T2*-weighted fMRI images in the axial plane. The acquired image volume consisted of $32 \times 3$-mm-thick slices, which allowed us to image the entire cortex, apart from the most ventral parts of the temporal lobe. Two blocks of 350 images [with an interscan interval (TR) of $3.5 \mathrm{sec}$ ] were acquired, with 70 images for each of the five conditions, giving a total scanning time of $\sim 41$ $\min (2 \times 20.5 \mathrm{~min}$ blocks $)$. Each condition was presented for the duration of seven scans $(24.5 \mathrm{sec})$. Experimental conditions were presented 10 times each in a counterbalanced manner (both within and between subjects). A structural MRI image also was acquired (using a standard T1-weighted scanning sequence) to allow for anatomically specific localization of significant areas of brain activation.

\section{Data analysis}

Behavioral data. RT data for each of the attentional tasks performed before scanning and during PET scanning were analyzed by repeated measures ANOVAs. The value of informative cues was assessed in an ANOVA that compared the four task conditions (ST, S, T, N), of which $\mathrm{N}$ provided noninformative cues, whereas all other conditions provided valid cue information for $80 \%$ of the trials. The relative advantage and disadvantage for valid and invalid cues was assessed with an ANOVA on data from the two attention conditions in which cueing information was provided along one dimension only (i.e., $\mathrm{S}$ and $\mathrm{T}$ ). The ANOVA tested for the effect of task condition (S, T), cue validity (valid, invalid), target side (left, right), and interval duration (short, long). A final ANOVA looked at data from the attention condition that used both spatially and temporally informative cues simultaneously (ST) and tested the effects of cue validity, target side, and interval duration.

PET and fMRI data. All functional images (either PET images or $\mathrm{T} 2 *$-weighted fMRI images) for each subject were realigned to the first image to correct for head movement between scans. Then the structural MRI was coregistered to the functional images to put both functional and structural images into the same space. All images were spatially normalized into a standard space (Talairach and Tournoux, 1988) by matching each image to a standardized MNI template (Montréal Neurological Institute, Québec, Canada), using both linear and nonlinear three-dimensional transformations (Friston et al., 1995a). Functional images were smoothed to accommodate intersubject differences in anatomy, using isotropic Gaussian kernels of 16 and $8 \mathrm{~mm}$ for the PET and fMRI data, respectively, which yielded a final resolution of $16 \times 17 \times 18$ $\mathrm{mm}$ at full-width half-maximum for the PET data and $12 \times 12 \times 11 \mathrm{~mm}$ for the fMRI data. The difference in degree of smoothing was reflective of the anatomical resolution of each scanning technique.

Functional images were analyzed with statistical parametric mapping (Friston et al., 1991) (SPM97d, Wellcome Department of Cognitive Neurology, London, UK). Condition and subject effects were estimated according to the general linear model at each voxel in brain space (Friston et al., 1995b). For the PET data, indices of global blood flow were modeled as a confounding covariate (normalized to $50 \mathrm{ml}$ per 100 $\mathrm{ml} / \mathrm{min}$ ), using ANCOVA (Friston et al., 1990). For the fMRI data, repeated measures (scans) were collapsed within subject (adjusting for both global blood flow by using proportional scaling and low-frequency physiological drifts by using a high-pass filter of $300 \mathrm{sec}$ ) to give one scan per condition per subject. Then these conditions were compared between subjects, thereby effecting a random effects model, allowing inferences to be made about the general population. Analysis of single subjects also was performed to aid anatomical localization of significant activations from the group analysis.

For PET data, linear contrasts were used to test hypotheses about regionally specific condition effects, which produced a statistical parametric map of the $t$ statistic generated for each voxel (SPM $\{t\})$. The $\operatorname{SPM}\{t\}$ was transformed to a map of corresponding $Z$ values, thresholded at a $Z$ value of 3.09 ( $p=0.001$, uncorrected for multiple comparisons), and the resulting foci were characterized in terms of both spatial extent and peak height. The significance of each region corrected for multiple comparisons was estimated by using distributional approximations from the theory of Gaussian fields. For the fMRI data, a Gaussian temporal smoothing kernel of $6 \mathrm{sec}$ (at full-width half-maximum) was applied to the data during statistical analysis.

Statistical analysis was aimed at identifying common regions of activation for both spatial and temporal orienting as well as regions that were involved selectively in each type of attentional cueing. Common areas
Table 1. Reaction times (msec) (and SE) for validly cued targets in the $\mathrm{S}, \mathrm{T}$, and ST conditions and for all targets in the $\mathrm{N}$ condition

\begin{tabular}{ll} 
Condition & RT \\
\hline S & $322.11(8.4)$ \\
T & $315.54(13.0)$ \\
ST & $298.83(8.2)$ \\
N & $331.31(10.6)$
\end{tabular}

were defined by the statistical conjunction (Price and Friston, 1997) of the comparisons between each attentional cueing condition ( $\mathrm{S}$ and $\mathrm{T}$ ) to the resting baseline. Rest was chosen as an appropriate baseline for two reasons. First, this comparison provides the most compatibility with previous reports in the literature by using visual fixation controls (Corbetta et al., 1993). Second, although the neutral condition would seem like a superior alternative, this condition in itself engages attention and orients it along two spatial locations and two temporal intervals. The comparisons that use the resting baseline therefore provide the fuller picture of brain areas involved in attentional orienting. However, brain areas involved in general perceptual and motor demands of the task also will be revealed in these comparisons. Therefore, we also computed common areas for spatial and temporal orienting, using the neutral condition $(\mathrm{N})$ as a baseline, to identify areas that were involved specifically in both focused spatial and focused temporal orienting. Both of these conjunctions were computed by showing only the significant areas of activation for one comparison (spatial orienting vs baseline) that also were activated significantly in the other comparison (temporal orienting vs baseline). To preserve the orthogonality of these comparisons, we divided the baseline scans equally between the two contrasts. We used a significance threshold that was uncorrected for multiple comparisons, because the neural correlates of attentional orienting already have been well defined (Nobre et al., 1997), enabling us to make clear predictions about the areas we expected to be activated.

Brain regions selectively involved in spatial attention were obtained by contrasting the two conditions in which the cues contained spatial information (S and ST) with the two conditions that did not ( $\mathrm{T}$ and $\mathrm{N}$ ) (i.e., the main effect of space in the factorial design). To avoid potential contributions from deactivations linked to temporal orienting, we interrogated only those brain regions that also were activated significantly more by spatial orienting than by rest [i.e., the $\mathrm{S}$ minus Rest contrast $(p<0.001)$ was used to mask the entire brain volume]. Brain regions selectively involved in temporal orienting were obtained in an analogous manner [the main effect of time masked by T minus Rest $(p<0.001)$ ]. Brain regions preferentially engaged when attention was oriented in both time and space simultaneously, as opposed to either one separately, also were calculated (ST minus S and T, masked by ST minus Rest).

\section{RESULTS}

\section{Behavioral data}

Behavioral results are reported from both PET and fMRI subjects pooled together $(n=12)$. Data from two of the fMRI subjects and one of the PET subjects were lost because of technical difficulties. Separate analyses for PET and for fMRI subjects yielded equivalent effects, suggesting that each of the subject samples is representative of the larger group.

The comparison of RTs across all task conditions (Table 1) revealed that RTs were significantly faster when informative cues were provided than when neutral cues were used $\left[F_{(3,33)}=5.25\right.$; $p=0.005$; paired $t$ test comparing $\mathrm{N}$ with all other conditions $\left.t_{(11)}=2.68 ; p=0.02\right]$. RT data in the tasks with cues that were informative in one dimension ( $\mathrm{S}$ and $\mathrm{T}$ ) showed a significant main effect of task $\left[F_{(1,11)}=11.23 ; p<0.01\right]$, such that the $\mathrm{T}$ task was performed significantly faster $(320 \mathrm{msec})$ than the $\mathrm{S}$ task (352 msec) (Table 2). There was also a significant main effect of validity $\left[F_{(1,11)}=35.55 ; p<0.001\right]$. Responses were significantly faster in valid trials $(319 \mathrm{msec})$ as compared with invalid trials (363 msec). These validity effects were confirmed in separate 


\begin{tabular}{|c|c|c|c|c|c|}
\hline Condition & Validity & Left/short & Left/long & Right/short & Right/long \\
\hline \multirow[t]{2}{*}{ S } & V & $323.55(12.8)$ & $318.40(10.5)$ & $319.31(10.3)$ & 327.17 (11.2) \\
\hline & I & $383.44(12.4)$ & $375.59(13.2)$ & 361.54 (12.7) & 410.75 (17.3) \\
\hline \multirow[t]{2}{*}{$\mathrm{T}$} & V & $313.16(12.2)$ & $310.62(13.8)$ & $314.20(12.6)$ & 324.17 (18.0) \\
\hline & I & $369.99(17.4)$ & $331.96(20.9)$ & $354.50(15.5)$ & $319.48(10.2)$ \\
\hline \multirow[t]{2}{*}{ ST } & V & $301.28(10.7)$ & 297.15 (11.4) & $304.62(9.64)$ & $292.28(10.2)$ \\
\hline & I & $365.58(19.0)$ & $345.86(18.2)$ & $340.20(15.4)$ & $340.61(15.8)$ \\
\hline $\mathrm{N}$ & V & $328.63(12.9)$ & 331.69 (14.8) & $326.93(12.4)$ & $343.38(21.1)$ \\
\hline
\end{tabular}

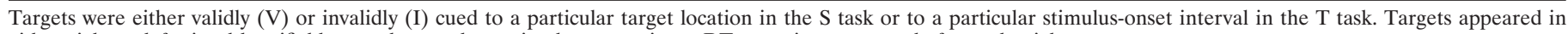
either right or left visual hemifield or at short or long stimulus-onset times. RTs are given separately for each trial type.

analyses of the $\mathrm{S}$ and $\mathrm{T}$ conditions individually [condition $\mathrm{S}$ : $F_{(1,11)}=26.60, p<0.001 ;$ condition T: $\left.F_{(1,11)}=12.78, p<0.005\right]$. Significant interactions between task and validity $\left[F_{(1,11)}=5.78\right.$; $p<0.05]$ and between task and duration $\left[F_{(1,11)}=15.41 ; p<\right.$ 0.005 ] also were observed. These were qualified by a three-way interaction among task, validity, and duration $\left[F_{(1,11)}=7.28 ; p<\right.$ $0.05]$. This reflected a large validity effect in all conditions except those in which temporal cues in the $\mathrm{T}$ condition incorrectly predicted the target's appearance at the long time interval (i.e., there was very little deleterious effect when the subject expected the target to occur at the short time interval, but it actually occurred at the longer one) (see Table 2). In the condition with informative cues along two dimensions (ST), a main effect of cue validity was obtained $\left[F_{(1,11)}=29.26 ; p<0.001\right]$, with valid RTs (299 msec) being faster than invalid ones (348 msec). Full behavioral data are summarized in Table 2.

\section{PET data}

\section{Common activations for spatial and temporal orienting}

Resting baseline. The spatial and temporal orienting tasks activated many brain regions in common. The common network of brain regions was defined by the conjunction of the comparisons between each attentional cueing condition ( $\mathrm{S}$ and $\mathrm{T}$ ) to the resting baseline. Table 3 and Figure 2 present the brain areas that were activated in common by the spatial and temporal orienting tasks. Frontal areas of the network included the lateral premotor cortex bilaterally (BA 6), which extended medially and dorsally toward the supplementary motor area (BA 6). These locations are consistent with previous reports of frontal eye fields (FEF) (Paus, 1996). Posterior parietal activations also were obtained bilaterally. In both hemispheres, activations occurred in the intraparietal sulcus and extended into the inferior parietal lobule (BA 40). An additional separate cluster of activation occurred in the right hemisphere only, centered on the intraparietal sulcus at a more posterior and superior location. Large clusters of activations were centered over ventral visual cortical areas, particularly of the left hemisphere. Bilateral cerebellum also was activated.

Neutral condition baseline. There were fewer areas activated in common when the neutral condition was used as a baseline. Of the regions activated in common and using rest as the baseline, only the left ventral posterior visual cortex $(x, y, z=-54,-74$, $-8 ; Z=3.58 ; p<0.001$ ) was common to both spatial and temporal orienting. A subthreshold activation in the left parietal cortex also was observed $(x, y, z=-58,-48,46 ; Z=2.85$; $p=0.002)$.

\section{Spatial orienting}

Spatial orienting of attention was associated with significantly more activation in the right posterior parietal cortex in the
Table 3. Common areas of activation for spatial and temporal orientation of attention as measured by PET

Brain areas $x y z(\mathrm{~mm})$ $Z$ score

a. Frontal cortex

1) $R$ lateral premotor (BA 6)

2) $R$ medial promotor (BA 6)

3) $R$ ventrolateral prefrontal (BA 11)

4) L lateral motor/premotor (BA 4/6)

5) L medial premotor (BA 6)

6) L ventrolateral prefrontal (BA 11)

$\begin{array}{lll}46,0,42 & 5.67 & <0.001 \\ 8,6,58 & 2.89 & <0.001 \\ 28,52,-22 & 3.48 & <0.001 \\ & & \\ -34,-4,44 & 5.29 & <0.001 \\ & & <0.001 \\ -4,-4,68 & 4.64 & <0.001 \\ -26,32,-32 & 3.86 & \end{array}$

\section{b. Parietal cortex}

7) $\mathrm{R}$ inferior parietal lobule $\quad 66,-44,34 \quad 3.23=0.001$ (BA 40) and intraparietal 58, $-40,40 \quad 2.79 \quad=0.003$ sulcus

8) $\mathrm{R}$ posterior intraparietal $\quad 38,-64,52 \quad 3.11 \quad=0.001$ sulcus

9) L inferior parietal lobule (BA 40) and intraparietal $-44,-42,30 \quad 4.22 \quad<0.001$ sulcus

$-38,-50,46 \quad 3.56 \quad<0.001$

\section{c. Visual cortex}

10) $R$ ventral posterior visual $\quad 42,-80,-10 \quad 3.53 \quad<0.001$

11) $\mathrm{L}$ ventral posterior visual $\quad-46,-64,6 \quad 4.80 \quad<0.001$ $-42,-86,-10 \quad 4.57 \quad<0.001$

$\begin{array}{llll}\text { d. } \text { Subcortical } \\ \text { L putamen } & -24,0,2 & 3.23 & <0.001 \\ & & & \\ \text { R cerebellum } & 42,-80,-26 & 4.26 & <0.001 \\ \text { L cerebellum } & -34,-58,-40 & 4.42 & <0.001\end{array}$

$p$ values are uncorrected for multiple comparisons.

inferior parietal lobule (Table 4a). This focus was within the general area of common right parietal activations across types of orienting (see Table 3). Activation in the right inferior parietal lobule was, therefore, significantly higher in the spatial cueing condition relative to temporal cueing but was not involved exclusively in spatial cueing. Figure $3 A a$ shows the inferior parietal activation rendered on a surface view of a standard brain and on a sagittal section of the averaged MRI of all seven subjects. Activations also were found in the left ventral visual cortex and cerebellum. 


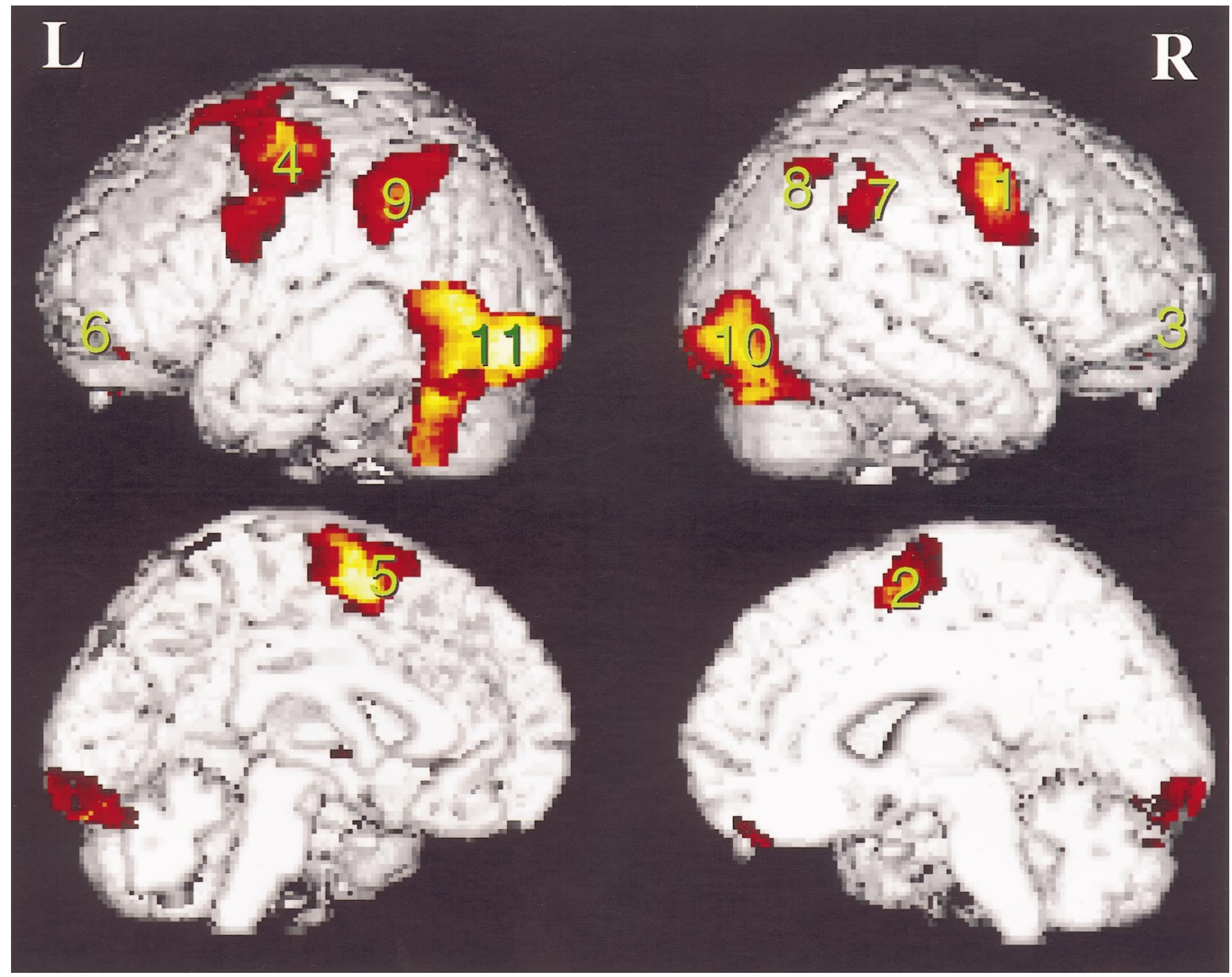

Figure 2. Areas commonly activated by spatial and temporal orienting of attention, as measured by PET. Activations are numbered according to Table 3 .

\section{Temporal orienting}

Brain regions specifically correlated with temporal orienting are presented in Table $4 b$. Two brain areas within the common network of brain areas (see Table 3) were activated significantly more for the temporal, than for the spatial, orienting condition: the left intraparietal sulcus and the left cerebellum. In addition, the left ventral premotor cortex in the general region of Broca's area (BA 6/44) was activated. The latter region was not part of the common network and suggests a more exclusive relationship to aspects of temporal orienting of attention. The location of the brain activations selective for temporal orienting are shown in Figure $3 A b$, rendered on a surface view of a standard brain. The left premotor area is shown on a sagittal section of the averaged MRI of all seven subjects.

\section{Combined spatiotemporal orienting}

The interaction between spatial and temporal orienting isolated the areas that additionally were activated by attending to both spatial and temporal cues simultaneously, as opposed to either one separately. The resulting activations occurred within parietal cortex and are summarized in Table $4 c$. Activations were obtained around the intraparietal sulcus bilaterally in regions that overlapped with the common network (see Table 3). In addition, the inferior region of the right temporoparietal junction, which straddled both inferior parietal and posterior superior temporal cortices, was activated exclusively by combined spatial and temporal orienting. The locations of the brain activations selective for combined spatial and temporal orienting are shown in Figure $3 A c$, rendered on a surface view of a standard brain. The bilateral intraparietal sulcus activations are shown on two coronal sections of the averaged MRI of all seven subjects.

\section{fMRI data}

\section{Common activations for spatial and temporal orienting}

The spatial and temporal orienting tasks activated many brain regions in common. The common network of brain regions was defined by the conjunction of the comparisons between each attentional cueing condition ( $\mathrm{S}$ and $\mathrm{T}$ ) to the resting baseline. Table 5 presents the brain areas that were activated in common by the spatial and temporal orienting tasks and is strikingly similar to those areas activated by the similar comparison in the PET study. Frontal areas of the network included the lateral premotor cortex bilaterally (BA 6), which extended medially and dorsally toward the supplementary motor area (BA 6), and ventral frontal cortex 


\begin{tabular}{|c|c|c|c|}
\hline Brain areas & $x y z(\mathrm{~mm})$ & $Z$ score & $p$ \\
\hline \multicolumn{4}{|l|}{ a. Spatial orienting } \\
\hline \multirow[t]{2}{*}{$\mathrm{R}$ inferior parietal lobule (BA 40) } & $62,-46, \quad 32$ & 3.67 & $<0.001$ \\
\hline & $58,-44, \quad 42$ & 3.47 & $<0.001$ \\
\hline L occipital-temporal sulcus & $-48,-70,-12$ & 3.48 & $<0.001$ \\
\hline L cerebellum & $-24,-50,-46$ & 3.30 & $<0.001$ \\
\hline \multicolumn{4}{|l|}{ b. Temporal orienting } \\
\hline \multirow[t]{2}{*}{$\mathrm{L}$ intraparietal sulcus } & $-42,-48, \quad 48$ & 3.33 & $<0.001$ \\
\hline & $-44,-44, \quad 38$ & 3.29 & $=0.001$ \\
\hline \multicolumn{4}{|l|}{ L lateral inferior premotor } \\
\hline (BA 6/44) & $-44, \quad 4, \quad 20$ & 3.42 & $<0.001$ \\
\hline L cerebellum & $-48,-68,-24$ & 3.25 & $=0.001$ \\
\hline \multicolumn{4}{|l|}{$\begin{array}{l}\text { c. Combined spatial and temporal } \\
\text { orienting }\end{array}$} \\
\hline \multicolumn{4}{|l|}{$\mathrm{R}$ temporal-parietal junction } \\
\hline (BA 39/40) & $68,-46, \quad 22$ & 3.29 & $<0.001$ \\
\hline $\mathrm{R}$ intraparietal sulcus & $38,-64, \quad 40$ & 3.05 & $<0.001$ \\
\hline $\mathrm{L}$ intraparietal sulcus & $-50,-40, \quad 48$ & 2.98 & $=0.001$ \\
\hline
\end{tabular}

$p$ values are uncorrected for multiple comparisons.

bilaterally. Intraparietal sulcus activations also were obtained bilaterally. Large clusters of activations were centered over ventral visual cortical areas, extending into the cerebellum in both hemispheres.

Neutral condition baseline. There were fewer areas activated in common when the neutral condition was used as a baseline. Of the regions activated in common and using rest as the baseline, only the left parietal cortex $(x, y, z=-45,-30,39 ; Z=3.01 ; p=$ 0.001 ) was common to both spatial and temporal orienting.

\section{Spatial orienting}

Spatial orienting of attention was associated with significant activation of the right intraparietal sulcus, extending down toward the temporoparietal junction, and a similar, although weaker, activation in the left intraparietal sulcus (Table $6 a$ ). The right visual cortex and bilateral cerebellum also were activated. Figure $3 B a$ shows the precise location of the right intraparietal sulcus activation in three of the eight subjects.

\section{Temporal orienting}

Temporal orienting of attention produced focal activation of the left intraparietal sulcus and left cerebellum only (Table 6b). Figure $3 B b$ shows the location of the left intraparietal sulcus activation in three of the eight subjects.

\section{Combined spatiotemporal orienting}

The interaction between spatial and temporal processing isolated areas that were activated additionally by attending to both spatial and temporal cues simultaneously, as opposed to either one separately. The resulting activations occurred within the intrapa- rietal sulcus bilaterally and cerebellum bilaterally (Table $6 c$ ). Figure $3 B c$ shows the location of the bilateral intraparietal sulcus activation in three of the eight subjects.

\section{DISCUSSION}

We have demonstrated a behavioral advantage of knowing when to expect a stimulus to occur, which is qualitatively similar to that for knowing where to expect it to occur. Although the benefits of spatial cueing are well established, this is the first demonstration that such benefits also can manifest themselves when attention is paid to moments in time. One exception is the study by Kingstone (1992), which investigated the interaction between attending to form and attending to temporal interval but did not investigate temporal cueing per se. Convergent findings from our own PET and fMRI studies indicated a considerable overlap between neural networks for performing spatial or temporal orienting of attention tasks but also showed functional specialization in certain brain regions. The PET experiment defined the networks for spatial and temporal orienting. The fMRI experiment replicated the results and additionally improved anatomical localization by individual subject analysis. Specifically, the left intraparietal sulcus (IPS) and the left inferior premotor cortex (BA44/6) were involved in attention to time intervals. In contrast, the right IPS was activated during spatial orientation, confirming earlier functional imaging (Corbetta et al., 1993; Nobre et al., 1997) and neuropsychological studies (Posner et al., 1984).

\section{Behavioral advantages of attending to time}

Attention to temporal intervals is a relatively novel concept, which represents how directing attentional resources to specific moments in time can influence behavior. This is directly analogous to the concept of spatial orienting (Posner et al., 1980) whereby shifting attention to a likely target location improves signal detection. Indeed, subjects drawn from two independent samples demonstrated RT benefits in all conditions when cues were spatially or temporally informative, as compared with neutral cues. Furthermore, subjects showed behavioral costs for invalid, as compared with valid, trials in both spatial and temporal orienting. An interaction among task, validity, and stimulus-onset time suggested that the behavioral cost was smaller for targets presented at long intervals in the temporal condition. Because of the bimodal nature of stimulus-onset times, omission of the target at the short interval guaranteed it would occur at the long interval (see also Kingstone, 1992). Subjects then could reorient attention to the later time point.

\section{Common neural network for spatial and temporal orienting of attention}

A number of brain regions were activated by both spatial and temporal orienting tasks, as compared with a resting baseline, suggesting the existence of a ubiquitous system for allocating attentional resources in general, independent of stimulus dimension. The areas were consistent with theoretical proposals of a frontoparietal attentional network, based on neuropsychological evidence (Mesulam, 1981, 1990; Kinsbourne, 1987; Rizzolatti et al., 1987; Posner and Petersen, 1990). Our results extend these theories by showing that these brain areas are recruited not just for the orienting of spatial attention but more generally for other stimulus dimensions as well. Furthermore, the specific areas recruited (IPS and FEF) were consistent with those observed in oculomotor tasks, thus supporting a strong relationship between oculomotor preparation and attentional orienting (Rizzolatti et 
A

(a)

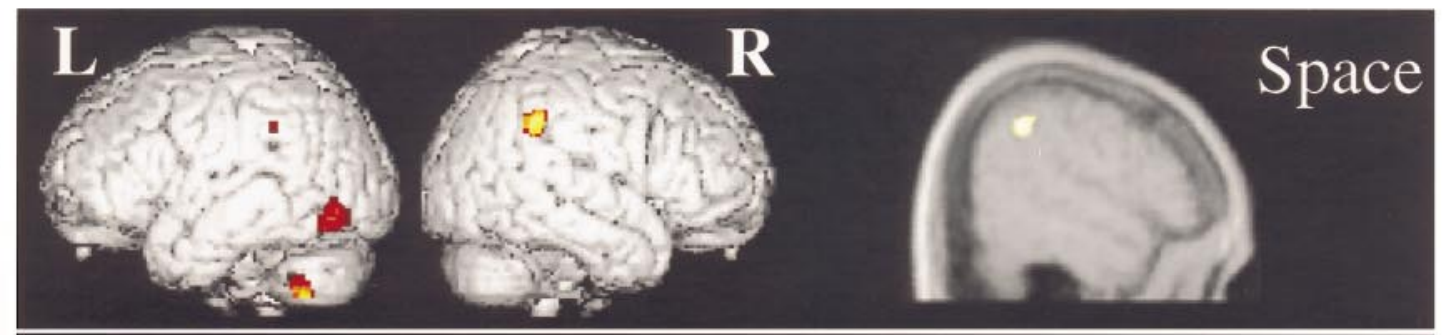

(b)

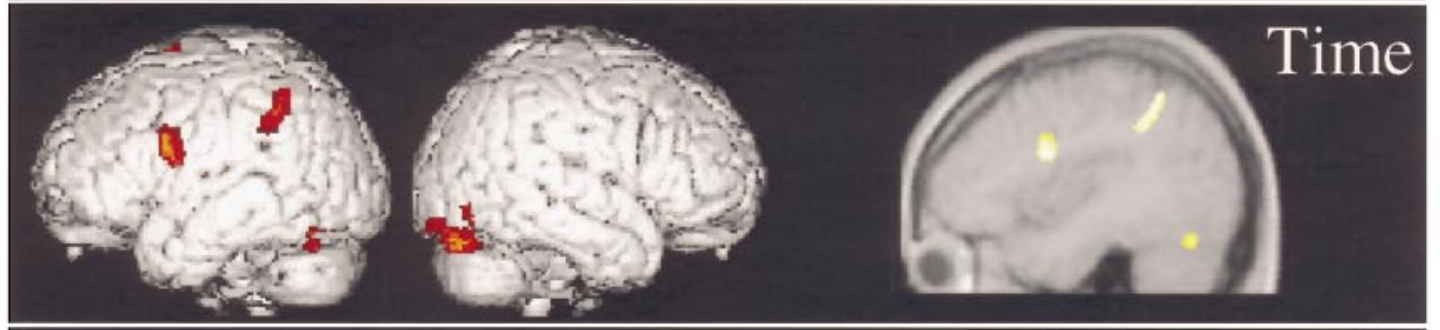

(c)

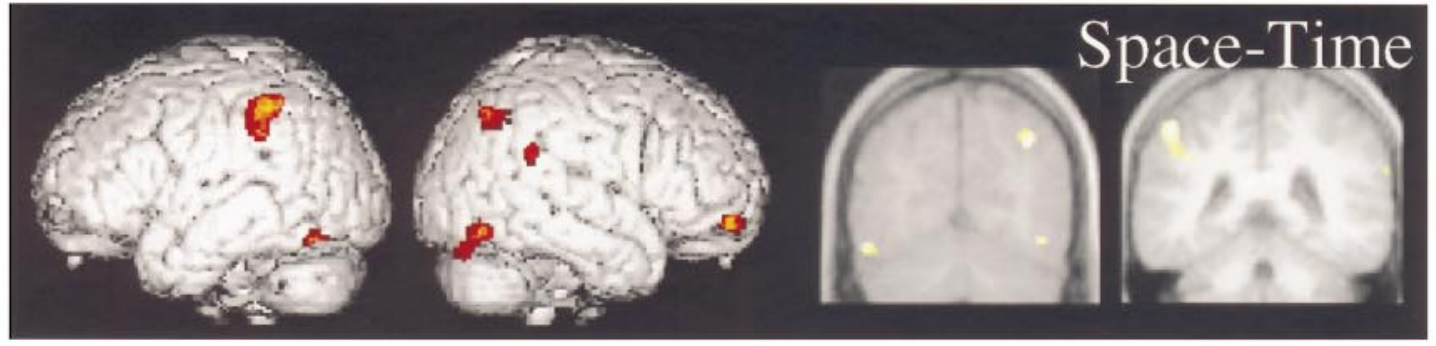

(a) $\mathrm{S}$

(b)

B

S1

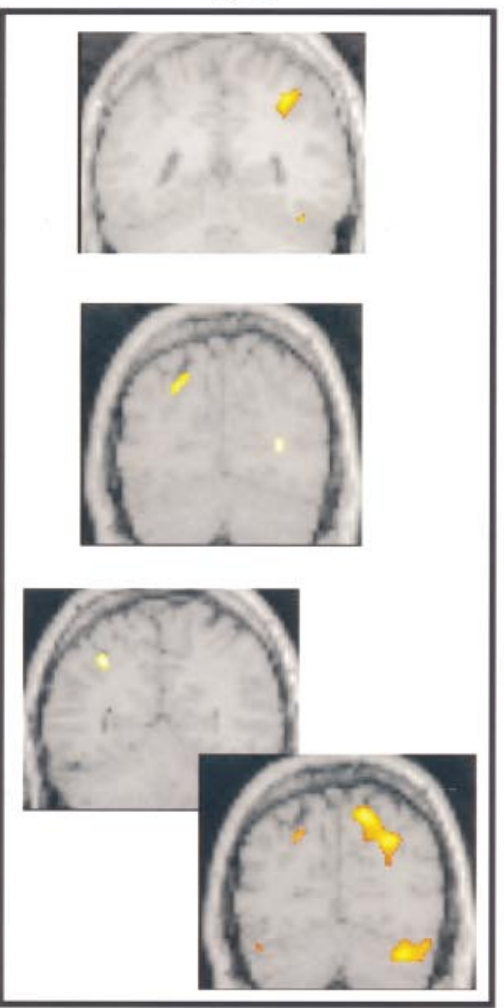

S2
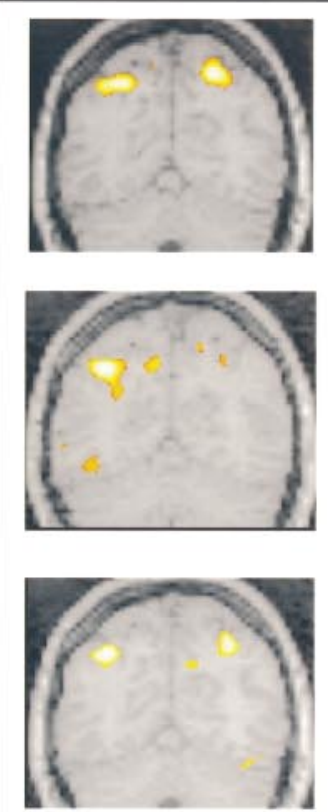

S3

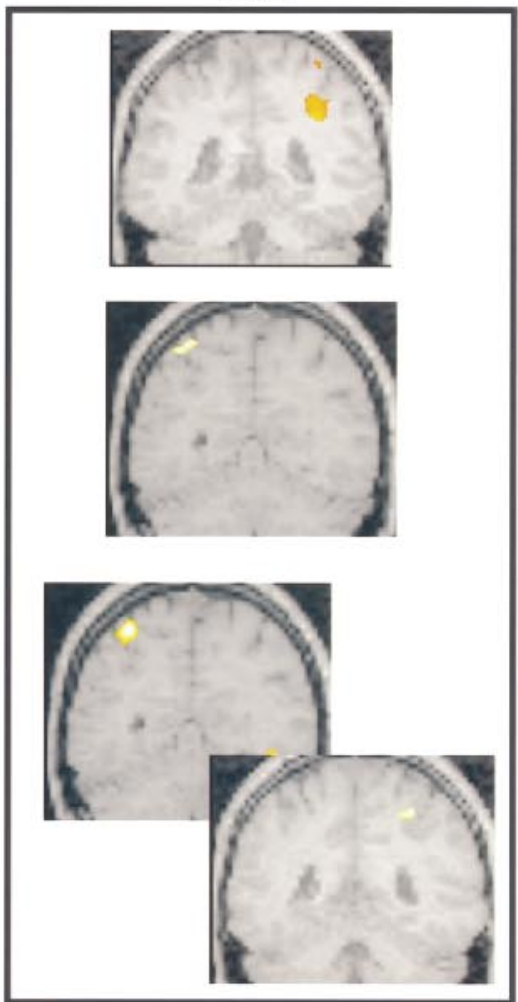

Figure 3. A, Areas preferentially activated by $(a)$ spatial orienting, $(b)$ temporal orienting, and $(c)$ spatiotemporal orienting of attention, as measured by PET. $B$, Anatomical localization of the preferential activation of $(a)$ the right intraparietal sulcus by spatial orienting $(S)$, $(b)$ the left intraparietal sulcus by temporal orienting $(T)$, and $(c)$ the bilateral intraparietal sulcus by spatiotemporal orienting $(S T)$, as measured by fMRI. Coronal slices from three separate individuals $(S 1, S 2, S 3)$ are shown for each condition. The left side of the figure corresponds to the left side of the brain. 


\begin{tabular}{|c|c|c|}
\hline Brain areas & $x y z(\mathrm{~mm})$ & $Z$ score \\
\hline \multicolumn{3}{|l|}{ Frontal cortex } \\
\hline R lateral premotor (BA 6) & $48,-3,42$ & 6.47 \\
\hline $\mathrm{R}$ medial premotor (BA 6) & $9,0,57$ & 7.01 \\
\hline $\mathrm{R}$ dorsolateral prefrontal (BA 46) & $36,39,18$ & 5.39 \\
\hline $\mathrm{R}$ ventrolateral prefrontal (BA 47) & $42,47,-6$ & 6.56 \\
\hline $\mathrm{R}$ insula & $27,6,-3$ & 7.20 \\
\hline L lateral motor/premotor (BA 4/6) & $-51,0,39$ & 6.34 \\
\hline L medial premotor (BA 6) & $-3,0,51$ & 7.82 \\
\hline L dorsolateral prefrontal (BA 46) & $-36,48,24$ & 4.99 \\
\hline L ventrolateral prefrontal (BA 47) & $-30,27,-6$ & 5.47 \\
\hline $\mathrm{L}$ insula & $-24,9,0$ & 7.48 \\
\hline \multicolumn{3}{|l|}{ b. Parietal cortex } \\
\hline $\mathrm{R}$ intraparietal sulcus & $36,-54,45$ & 6.89 \\
\hline $\mathrm{L}$ intraparietal sulcus & $-42,-30,39$ & 7.23 \\
\hline \multicolumn{3}{|l|}{ c. Visual cortex } \\
\hline $\mathrm{R}$ ventral posterior visual & $39,-84,-9$ & 5.51 \\
\hline $\mathrm{L}$ ventral posterior visual & $-39,-72,-9$ & 6.96 \\
\hline \multicolumn{3}{|l|}{ d. Sucbcortial } \\
\hline $\mathrm{R}$ thalamus & $12,-9,3$ & 6.40 \\
\hline L thalamus & $-12,-12,0$ & 6.30 \\
\hline $\mathrm{R}$ cerebellum & $36,-87,-18$ & 6.56 \\
\hline L cerebellum & $-48,-60,-21$ & 6.70 \\
\hline
\end{tabular}

All areas are significant at a corrected threshold of $p<0.05$.

al., 1987; Corbetta, 1998; Nobre et al., 1998). When the neutral cue was used as a baseline for determining the overlap between the two attentional dimensions, very few brain areas were revealed. This indicates that the selective orienting of attention in time or space is subserved by nonoverlapping brain areas.

\section{Hemispheric lateralization for spatial and temporal orienting}

The right hemisphere bias for spatial orienting in this study is consistent with neuropsychological theories (Mesulam, 1981; Kinsbourne, 1987) and previous brain-imaging studies (Corbetta et al., 1993; Nobre et al., 1997). The left hemisphere bias for temporal orienting was a novel finding. This, coupled with the specific constellation of the brain regions involved, helps constrain hypotheses regarding how temporal orienting may be implemented in the brain and how it may affect ongoing stimulus processing. Temporal orienting may affect perceptual analysis of stimuli in a manner analogous to spatial orienting (see Mangun, 1995), may affect motor preparation or timing, or may affect both. Similar left-sided asymmetries have been reported for the processing of fine temporal discriminations and during motor preparation, suggesting that these processes may be involved in temporal orienting.

Platel et al. (1997) found activation of the left inferior frontal gyrus (BA 44/6) for attention to rhythm of sequences of notes. Rhythm perception requires the ability to process the temporal characteristics of sequential stimuli. Similarly, Fiez et al. (1995) found activation of the left frontal operculum in conditions that
Table 6. Selective areas of activation for distinct types of attentional orienting as measured by fMRI

\begin{tabular}{llll} 
Brain areas & $x y z(\mathrm{~mm})$ & $Z$ score & $p$ \\
\hline $\begin{array}{l}\text { a. Spatial orienting } \\
\text { R inferior parietal lobule (BA 40) }\end{array}$ & $48,-48,21$ & 3.69 & $<0.001$ \\
$\quad$ and intraparietal sulcus & $42,-36,33$ & 3.60 & $<0.001$ \\
& $36,-54,48$ & 2.99 & $=0.001$ \\
& & & \\
R cerebellum & $51,-69,-15$ & 3.79 & $<0.001$ \\
L cerebellum & $-51,-66,-18$ & 3.35 & $<0.001$
\end{tabular}

b. Temporal orienting

L intraparietal sulcus

$-24,-69,36 \quad 4.05 \quad<0.001$

L cerebellum

$-21,-72,45 \quad 3.24 \quad=0.001$

$\begin{array}{llll}\begin{array}{l}\text { c. Combined spatial and temporal } \\ \text { orienting }\end{array} & & & \\ \text { R inferior parietal lobule (BA 40) } & 57,-27,27 & 3.48 & <0.001 \\ \quad \text { and intraparietal sulcus } & 39,-42,36 & 4.31 & <0.001 \\ & 18,-60,45 & 3.05 & =0.001 \\ \text { L intraparietal sulcus (posterior) } & -24,-69,36 & 3.59 & =0.001 \\ \text { L intraparietal sulcus } & -39,-39,30 & 3.23 & <0.001 \\ & & & \\ \text { R cerebellum } & 48,-54,-18 & 3.61 & <0.001 \\ \text { L cerebellum } & -48,-60,-21 & 3.73 & <0.001 \\ \end{array}$

$p$ values are uncorrected for multiple comparisons.

required perception of rapid temporal modulations, whether the stimuli were linguistic or not, as compared with stimuli without rapid temporal transitions. In a companion experiment that used visually displayed letter stimuli, the left frontal opercular activity was increased for tasks requiring temporal, as compared with orthographic, judgments. Thus, forming internal representations of temporal duration is sufficient to activate this area. These results support a theoretical framework (Tallal et al., 1993) that explains the left hemisphere dominance for language as originating from the specialization of the left hemisphere for rapid temporal integration (Merzenich et al., 1996). In accordance with this view, we find preferential activation of the left hemisphere for temporal orienting. This may reflect fine discrimination of temporal intervals, necessary for focusing attention at specific moments in time.

Hammond (1982) suggested that low-level cognitive processes, such as timing, may be common to several higher-order ones such as language or motor preparation. Examination of these more elemental functions may be a profitable line of research for characterizing the functional specializations of the two hemispheres. A recent study has demonstrated that areas traditionally associated with speech also are associated with the internal representation of movement, specifically the left inferior premotor and left inferior parietal cortex (M. F. S. Rushworth, M. Krams, R. E. Passingham, unpublished observations). Preparation to make either right or left finger movements activated the left inferior premotor cortex (Broca's area), which is the same area recruited for speech preparation (Krams et al., 1998; M. F. S. Rushworth, M. Krams, R. E. Passingham, unpublished observations). This study used fixed intervals between cue and response, allowing the subjects to anticipate the moment at which they had 
to respond. A previous study by Deiber et al. (1996) found only left parietal cortex activations when the interval between cue and response was random. It may be that the left parietal activation relates to the type of movement to be made (see also Rushworth et al., 1997), whereas the left frontal activation relates more to when the movement should be made. This raises the possibility of a distinction between attention to a motor act and attention to a moment in time per se. Motor anticipation and preparation are likely to have played an important role in the attention effects in the temporal orienting condition of our own study. Further experiments are required to distinguish between anticipation of a motor act and attention to temporal intervals per se.

Recently, studies have begun to investigate nonspatial aspects of attention, using tasks with rapid, successive, foveal stimuli. Brain imaging (Coull et al., 1996; Wojciulik and Kanwisher, 1998) and neuropsychological studies (Harrington et al., 1998) have identified areas that overlap with those for spatial attention, such as the right posterior parietal cortex. Harrington et al. (1998) concluded from impairments in nonspatial attentional orienting in patients with right or left parietal lesions that "parietal cortex is essential for covert shifts of attention to temporal stimuli." Our results agree with findings from studies that used nonspatial attention tasks by demonstrating a general bilateral frontoparietal attentional orienting network across different stimulus dimensions. However, these nonspatial tasks are more likely to have measured short-term aspects of vigilance than to have measured attentional orientation to temporal intervals explicitly. Our own temporal orienting task measures selective attention to temporal intervals, and although both hemispheres were activated by the performance of this task, we demonstrated preferential activation of the left parietal (and inferior premotor) cortex. By contrast, using the attentional blink paradigm, Husain et al. (1997) have observed lapses in temporal attention in neglect patients with lesions of the right inferior parietal cortex (patients with left-sided lesions were not tested). However, this paradigm indexes the time course of the attentional process itself, whereas our own task represents the orienting of attention in time.

We note that temporal processing is not a unitary phenomenon and that it can be decomposed into many constituent parts. Hazeltine et al. (1997) suggest that this functional heterogeneity is reflected neuroanatomically, with different brain areas being involved in different aspects of timing. Our results suggest that a left frontoparietal network is recruited particularly for directing attention toward a particular moment in time.

\section{Parietal cortex and combined spatiotemporal orienting}

Simultaneous spatial and temporal attention activated mainly parietal areas. Parietal cortex, particularly on the right, was activated to an even greater extent when subjects directed their attention across both space and time together, as compared with either dimension alone. This area has been implicated in many aspects of attentional processing, such as spatial attention (Corbetta et al., 1993; Nobre et al., 1997) and sustained attention (Pardo et al., 1991). A recent PET study showed the right parietal cortex to be the main site of interaction between selective responding to nonspatial targets and sustained attention (Coull et al., 1998). Together, all of these findings raise the important possibility that the right parietal cortex not only is implicated in discrete forms of attention but also provides a site for one attentional process to influence, or interact with, another. Also notable was activation of the inferior parietal cortex, including the temporoparietal junction. This area has been implicated most con- sistently in clinical neglect (Rafal and Robertson, 1995) and may highlight the importance of temporal as well as spatial orienting to guide purposeful behavior.

In conclusion, we gathered behavioral and neuroimaging evidence supporting the existence of a ubiquitous neural network for attentional orienting. However, a striking hemispheric lateralization for attention to spatial location versus temporal interval also exists, with preferential activation of the right and left parietal areas for spatial versus temporal cues, respectively. Furthermore, modulation of different foci within parietal cortex across experimental conditions suggests functional heterogeneity of this region. Finally, we have found equivalent results by using both PET and fMRI scanning methodologies. This demonstrates the reliability of the task-specific activations and also cross-validates the sensitivity of each scanning technique.

\section{REFERENCES}

Corbetta M (1998) Frontoparietal cortical networks for directing attention and the eye to visual locations: identical, independent, or overlapping neural systems? Proc Natl Acad Sci USA 95:831-838.

Corbetta M, Miezin F, Shulman G, Petersen SE (1993) A PET study of visuospatial attention. J Neurosci 13:1202-1226.

Coull JT, Frith CD, Frackowiak RSJ, Grasby PM (1996) A frontoparietal network for rapid visual information processing: a PET study of sustained attention and working memory. Neuropsychologia 34:1085-1095.

Coull JT, Frackowiak RSJ, Frith CD (1998) Monitoring for target objects: activation of right frontal and parietal cortices with increasing time on task. Neuropsychologia, in press.

Deiber M-P, Ibanez V, Sadato N, Hallett M (1996) Cerebral structures participating in motor preparation in humans: a positron emission tomography study. J Neurophysiol 75:233-247.

Fiez JA, Raichle ME, Miezin FM, Petersen SE, Tallal P, Katz WF (1995) PET studies of auditory and phonological processing effects of stimulus characteristics and task demands. J Cogn Neurosci 7:357-375.

Friston KJ, Frith CD, Liddle PF, Dolan RJ, Lammertsma AA, Frackowiak RSJ (1990) The relationship between global and local changes in PET scans. J Cereb Blood Flow Metab 10:458-466.

Friston KJ, Frith C, Liddle P, Frackowiak R (1991) Comparing functional (PET) images: the assessment of significant change. J Cereb Blood Flow Metab 11:690-699.

Friston KJ, Ashburner J, Poline J-B, Frith CD, Heather JD, Frackowiak RSJ (1995a) Spatial registration and normalisation of images. Hum Brain Mapp 2:165-189.

Friston KJ, Holmes AP, Worsley KJ, Poline J-P, Frith CD, Frackowiak RSJ (1995b) Statistical parametric maps in functional imaging: a general linear approach. Hum Brain Mapp 2:189-210.

Hammond GR (1982) Hemispheric differences in temporal resolution. Brain Cogn 1:95-118.

Harrington DL, Haaland KY, Knight RT (1998) Cortical networks underlying mechanisms of time perception. J Neurosci 18:1085-1095.

Hazeltine E, Helmuth LL, Ivry RB (1997) Neural mechanisms of timing. Trends Cognit Sci 1:163-169.

Husain M, Shapiro K, Martin J, Kennard C (1997) Abnormal temporal dynamics of visual attention in spatial neglect patients. Nature 385:154-156.

Ivry RB, Keele SW (1989) Timing functions of the cerebellum. J Cogn Neurosci 1:136-152.

Jueptner IH, Rijntes M, Weiller C, Faiss JH, Timmann D, Mueller SP, Diener HC (1995) Localization of a cerebellar timing process using PET. Neurology 45:1540-1545.

Kingstone A (1992) Combining expectancies. Q J Exp Psychol 44[A]:69-104.

Kinsbourne M (1987) Mechanisms of unilateral neglect. In: Neurophysiological and neuropsychological aspects of spatial attention (Jeannerod M, ed). Amsterdam: Elsevier.

Krams M, Rushworth MFS, Deiber M-P, Frackowiak RSJ, Passingham RE (1998) The preparation, execution, and suppression of copied movements in the human brain. Exp Brain Res 120:386-398.

Mangun GR (1995) Neural mechanisms of visual selective attention. Psychophysiology 32:4-18.

Merzenich MM, Jenkins WM, Johnston P, Schreiber C, Miller SL, Tallal 
P (1996) Temporal processing deficits of language-learning impaired children ameliorated by training. Science 271:77-81.

Mesulam MM (1981) A cortical network for directed attention and unilateral neglect. Arch Neurol 10:304-325.

Mesulam MM (1990) Large scale neurocognitive networks and distributed processing for attention, language, and memory. Ann Neurol 28:597-613.

Nichelli P, Clark K, Hollnagel C, Grafman J (1995) Duration processing after frontal lobe lesions. Ann NY Acad Sci 769:183-190.

Nichelli P, Alway D, Grafman J (1996) Perceptual timing in cerebellar degeneration. Neuropsychologia 34:863-871.

Nobre AC, Gitelman DR, Sebestyen GN, Meyer J, Frackowiak RSJ, Frith CD, Mesulam MM (1997) Functional localisation of the system for visuospatial attention using positron emission tomography. Brain 120:515-533.

Nobre AC, Dias EC, Gitelman DR, Mesulam MM (1998) The overlap of brain regions that control saccades and covert visual spatial attention revealed by fMRI. Neuroimage 7:59.

Pardo JV, Fox PT, Raichle ME (1991) Localization of a human system for sustained attention by positron emission tomography. Nature 349:61-64.

Paus T (1996) Location and function of the human frontal eye field: a selective review. Neuropsychologia 34:475-483.

Platel H, Price C, Baron J-C, Wise R, Lambert J, Frackowiak RSJ, Lechevalier B, Eustache F (1997) The structural components of music perception: a functional anatomical study. Brain 120:229-243.

Posner MI, Petersen SE (1990) The attention system of the human brain. Annu Rev Neurosci 13:25-42.
Posner MI, Snyder CRR, Davidson BJ (1980) Attention and the detection of signals. J Exp Psychol Gen 109:160-174.

Posner MI, Walker JA, Friedrich FJ, Rafal RD (1984) Effects of parietal injury on covert orienting of attention. J Neurosci 4:1863-1874.

Price CJ, Friston KJ (1997) Cognitive conjunction: a new approach to brain activation experiments. NeuroImage 5:261-270.

Rafal R, Robertson L (1995) The neurology of visual attention. In: The cognitive neurosciences (Gazzaniga MS, ed), pp 625-648. Cambridge, MA: MIT.

Rao SM, Harrington DL, Haaland KY, Bobholz JA, Cox RW, Binder JR (1997) Distributed neural systems underlying the timing of movements. J Neurosci 17:5528-5535.

Raymond JE, Shapiro KL, Arnell KM (1992) Temporary suppression of visual processing in an RSVP task: an attentional blink? J Exp Psychol Hum Percept Perform 18:849-860.

Rizzolatti G, Riggio L, Dascola I, Umilta C (1987) Reorienting attention across the horizontal and vertical meridians-evidence in favor of a premotor theory of attention. Neuropsychologia 25:31-40.

Rushworth MFS, Nixon PD, Renowden S, Wade DT, Passingham RE (1997) The left parietal cortex and motor attention. Neuropsychologia 35:1261-1273.

Talairach J, Tournoux (1988) A co-planar stereotaxic atlas of the human brain. Stuttgart: Thieme.

Tallal P, Miller S, Fitch R (1993) Neurobiological basis of speech: a case for the preeminence of temporal processing. Ann NY Acad Sci 682:27-47.

Wojciulik E, Kanwisher N (1998) A general visual attention mechanism in the human brain: evidence from fMRI. Neuroimage 7:578. 